\title{
Desarrollos urbanos
} paralelos: Leonardo Da Vinci-Étienne Cabet

\section{Parallel urban developments: Leonardo $D a$} Vinci - Étienne Cabet

\section{Resumen:}

Arq. PhD. David Hidalgo

Autores:

García

dhidalgo@ugr.es

Arq. PhD. Juan Manuel

Santiago Zaragoza santi@ugr.es

Arq. Julián Arco Díaz juliannn@ugr.es

Departamento de Expresión Gráfica Arquitectónica y en

la Ingeniería

Universidad de Granada

España

Recibido: 19 Dic 2014 Aceptado: 17 Feb 2015
$\mathrm{L}$

eonardo da Vinci (1452-1519) encarna el ideal humanista del saber universal y es considerado como uno de los artistas y genios del Renacimiento. Es más conocido como pintor aunque realizó excelentes aportaciones a la arquitectura, a la morfología urbana y a la restauración. Su estudio de mayor importancia, en el campo de los estudios urbanos, es Cittá idéale donde expone la organización de una nueva ciudad que rompe con los sistemas urbanos tradicionales; esta obra presenta grandes similitudes con la ciudad utópica de Icara, descrita por Étienne Cabet (1788-1856) en su novela Voyage en Icarie publicada en París en 1840. Muestra las similitudes entre ambas propuestas publicadas en distintos ámbitos geográficos, con una diferencia de 355 años y cuyo único nexo es la ciudad de París.

Palabras clave: Leonardo, morfología urbana, París, Renacimiento, utopía.

\section{Abstract:}

Leonardo da Vinci (1452-1519) personifies the ideal humanist of the universal knowledge. He is also considered to be as one of the Renaissance geniuses and artists. His most known dimension is that of a painter, although to a lesser extent he contributed excellently to the fields of architecture, urban morphology and rehabilitation. His greatest study within the urban studies is his cittá idéale where he exposes his idea of a new city breaking with the traditional urban models. This one presents similarities with that of the utopic city of Icaria, described by the writer Étienne Cabet (1788-1856), in his novel titled Voyage en Icarie and published in Paris in1840. The current article describes the actual resemblances between both proposals, published in different worlds with a difference of 355 years and with only a connection which is the city of Paris.

Keywords: Leonardo, Paris, Renaissance, urban morphology, utopia. 


\section{Introducción}

Leonardo da Vinci es considerado como un excepcional artista y genio del Renacimiento y quizá de toda la historia de la humanidad. Su actividad profesional más conocida es la de pintor, pero también trabajó como inventor, anatomista, escultor, filósofo y escritor. Menos estudiadas resultan sus aportaciones en los campos de la arquitectura, la morfología urbana y la restauración; no por ello, de menor importancia.

Los motivos que justifican esta circunstancia son, en especial, dos: la inexistencia de evidencias prácticas de este tipo de trabajos y porque nunca cursó estudios oficiales. No obstante, su interés por los problemas y soluciones arquitectónicas y urbanísticas se aprecia en sus códices y manuscritos; posiblemente, entre todos sus estudios relacionados con la morfología urbana, su propuesta de Cittá idéale, con dos niveles de circulación, suscite mayor asombro entre los estudiosos de la materia y sea considerada como la más fascinante.

Fue diseñada tras la epidemia de peste que asoló la ciudad de Milán ${ }^{1}$ durante los años 1484-1485 y presentada a Ludovico Sforza con el objetivo de resolver "infra i popoli pieni d'infiniti mali" 2 (Firpo, 1963: 65). Circunstancia que le llevó a ser incluido dentro de la línea de tratadistas renacentistas que estudiaron y propusieron ciudades ideales, entre los que también se incluyen Antonio "Filarete", Francesco di Giorgio, Vincenzo Scamozzi, etc.

Étienne Cabet nació en Dijon-Francia y aunque inició sus estudios en Medicina, cambió rápidamente y se licenció en Derecho. En 1825 se trasladó a París donde tuvo una participación activa en la Revolución de 1830, que le supuso ser designado como miembro en la Cámara de Diputados. En 1834 fundó el periódico Le populaire con el objetivo de difundir sus ideas sobre política y Estado. ${ }^{3}$ Debido a que sus publicaciones se dirigían contra el Gobierno, tuvo

\footnotetext{
${ }^{1}$ La epidemia disminuyó en $2 / 3$ la población de la ciudad de Milán, convirtiéndose en un problema político, social y económico. Cantone indica: "La pressione demografica, I eccesiva densità edilizia, I inquinamento dell aria e delle acque, tutte cause di epidemie mortale" / "La presión demográfica, la excesiva densidad urbana y la contaminación del aire y del agua, todas causas de epidemias mortales" (Cantone, 1978: 70) [traducción de los autores].

2 "Los pueblos llenos de infinitos males" [traducción de los autores]. "Il Pacioli afierma che Leonardo aveva in progetto un trattato sull'architettura, di cui, probabilmente, avrebbero dovuto far parte le note gli schizzi "per una città ideale" contenuti nel Codice B" / "Pacioli indica que Leonardo había planeado un
}

que exiliarse a Inglaterra donde conoció al utopista Robert Owen y su programa contra la pobreza. ${ }^{4}$ Fue tal la impresión que le causó que a su regreso a Francia en 1840, publicó Voyage a Icaria. Esta novela que trata sobre la organización y desarrollo de una sociedad ideal, le reportó una importante fama y le supuso ser incluido dentro de los utopistas del siglo XIX, entre los que ya destacaban Robert Owen (1771-1858), Charles Fourier (1772-1837) y, posteriormente, Jean Baptiste Godín (1817-1889).

Los utopistas eran considerados hombres de la cultura del siglo XIX que desconfiaban de la ciudad industrial y proponían empezar de nuevo en otros lugares (Benévolo, 2007). Para ellos, el desarrollo que experimentaron las urbes durante la Revolución Industrial era un cúmulo de despropósitos que no permitía la restitución del orden y la disciplina de las épocas anteriores. Preferían la ciudad ideal a la real e intentaron materializarla y llegaron incluso a trasladar sus ideas a la práctica; gracias a ellos, el siglo XIX está considerado como "la edad de oro" de las ciudades utópicas (Choay, 1970: 125).

Tanto las propuestas de ciudades ideales de los tratadistas renacentistas como las de los utopistas del siglo XIX tenían como objetivos romper con la tendencia de desarrollo en las que se inscribían y mejorar las condiciones higiénicas de la población. Si las ciudades ideales pretendían solucionar los problemas de salubridad que se venían arrastrando desde la Edad Media, las propuestas utópicas del XIX perseguían urbes puras y limpias que se alejaran de los desmanes que se estaban produciendo en las ciudades industriales.

La elección de estos dos autores se debe a que sus propuestas presentan grandes similitudes, pese a existir entre ellas un lapso temporal de 355 años ¿Casualidad? La propuesta de Cittá idéale de Leonardo quedó reflejada en el Manuscrito identificado en el siglo XVIII por el Abad Giovan Battista Venturi, con la letra B (García, 2010). Este fue enviado en 1795, por orden de Napoleón Bonaparte, a la Biblioteca del Institut de France en

tratado de arquitectura en la que probablemente deberían aparecer los bocetos "para una ciudad ideal", que figuran en el 'Manuscrito B' (Cantone, 1978: 65) [traducción de los autores]. También lo afirma Heydenreich: "Tutta la tematica dell'architettura aveva in mente di ordinare e sistemare nella forma di un vero e proprio tratatto" / "Todo el tema de la arquitectura que tuvo en mente, estuvo ordenado y organizado en forma de un tratado. Toda la temática arquitectónica, que tenía en mente, ordenada y sistematizada en forma de auténtico tratado" (Heydenreich, 1972: 39) [traducción de los autores].

${ }^{3}$ En 1848 el periódico contaba con un tiraje de 5.000 ejemplares. ${ }^{4}$ La expuso por primera vez en 1817 , en un informe para la Comisión de Encuesta sobre la Ley de pobres (Benévolo, 2007). 
París, donde ha permanecido hasta la actualidad. Fue en esta Ciudad donde Étienne Cabet residió (1825-1835 /1840-1856) y publicó su obra Voyage a Icaria.

\section{Desarrollo}

Un análisis detallado de los trabajos e investigaciones de ambos autores permite observar las siguientes similitudes:

1. Ambos estudian la ciudad de forma global y proponen soluciones, ayudados por los avances en la innovación de la época.

Leonardo propone diez ciudades enlazadas entre sí y divididas en congregaciones: "E trarra 'di dieci città cinquemila case con trentamila abitazioni, e disgregherai tanta congregazione di popolo" 5 (Firpo, 1963: 66).

Son ciudades de nueva construcción, sin amurallar, atravesadas por un río que se incorpora a la trama urbana pues permite la circulación de barcas, con dos niveles de circulación, grandes espacios libres para esparcimiento de la población y un trazado con forma de retícula que se divide en congregaciones o "barrios". Una urbe que rompe con el estilo tradicional medieval de núcleo amurallado ${ }^{6}$ y trazado orgánico que se adelanta cuatrocientos años a las primeras tendencias en este tipo de ciudades.

De esta forma, la urbe de Leonardo asume un aspecto moderno y burgués propio del siglo XIX (véase Figura 1), con instalaciones de salubridad, espacios abiertos $y$ accesibles a las innovaciones técnicas de la época. En palabras del autor Luigi Firpo: "Si tratta di una cittò signoriale, una giustapposizione di palazzi nobiliari destinati a far corona ad una reggia principesca, o almeno un'accolta di residenze d'una borghesia agiata" 7 (Firpo, 1963: 80). También las palabras de Eugenio Garín señalan: "Città spaziosa, ampia, e quindi luminosa, resa viva dall acqua che non serve solo alle comunizacioni sui fiumi, all irrigazione con i canali all igiene, ma quasi ricongiunge alla natura, alla terra e al suo ritmo vitale" 8 (Garín, 1982: 315).

\footnotetext{
5 "Trazar diez ciudades de cinco mil casas con treinta mi habitantes y descomponerla en congregaciones del pueblo" [traducción de los autores].

${ }^{6}$ Las ciudades de la Edad Media eran urbes con una densidad de habitantes muy alta. El motivo de esta circunstancia era la existencia de una muralla de protección que encerraba un espacio limitado pero seguro. Como la ciudad no podía crecer en horizontal, lo hacía en vertical; es decir, en número de viviendas de altura. De esta forma se concentraba mayor población por superficie y por tanto la densidad era mayor. Pero este sistema defensivo era un elemento fundamental dentro de una sociedad
}

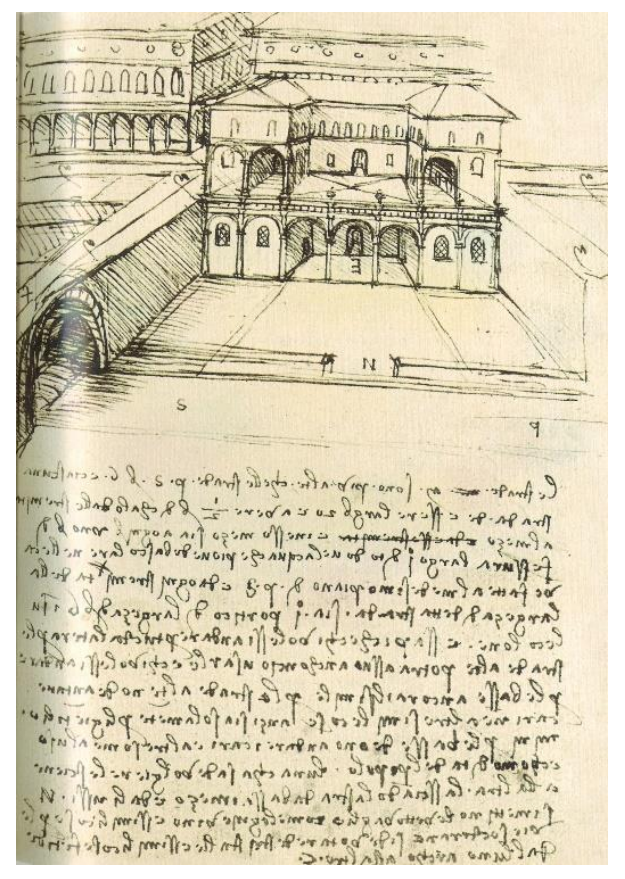

Figura 1: Aspecto de la propuesta Cittá idéale de Leonardo.

Fuente: Manuscrito B, folio 16r. Biblioteca Ambrosiana da Vinci.

Por su parte, Cabet define una gran metrópolis compuesta por sesenta barrios, atravesada por un río y con una distribución morfológica reticular. En su interior, aparecen una serie de espacios libres: la plaza central y plazas secundarias esparcidas por la trama urbana. El resto de manzanas están destinadas a viviendas mientras que la zona industrial, el cementerio y el hospital son concentrados en las afueras de la Ciudad, para eliminar las molestias que pueden generar a los habitantes. El propio Cabet la define como: "Es en realidad una descripción de la organización social y política de una comunidad; es un tratado científico y filosófico" (citado por Choay, 1970: 151).

Esta es una gran metrópoli atravesada por un río rectilíneo, construida sobre un plano perfectamente geométrico, con las calles en damero atravesadas por dos anillos de boulevard. La organización colectiva de la economía ha eliminado el comercio al por menor y, por tanto, las tiendas que son sustituidas por almacenes $y$ atelier estatales. Los cementerios, las fábricas y los

en lucha por el poder; por ello, su eliminación no era una opción a tener en cuenta.

7 "Se trata de una ciudad señorial, una yuxtaposición de palacios de la nobleza destinados a formar una corona o una región principesca, o al menos un grupo de residencias para una burguesía adinerada" [traducción de los autores].

8 "Ciudad espaciosa, amplia y, por tanto, luminosa, viva por el agua que no solo sirve para mantenerla comunicada a través de los ríos o para la irrigación mediante canales o para la higiene, sino que integra la naturaleza, la tierra y su ritmo vital" [traducción de los autores]. 
hospitales se encuentran fuera de la Ciudad en medio de espacios verdes. La circulación está regulada a fin de garantizar la seguridad de los peatones quienes recorren adecuados pasajes cubiertos, en tanto que los vehículos circulan por caminos apropiados de los cuales no pueden salirse, pues es obligatorio conservar la derecha. La Ciudad comprende sesenta barrios, cada uno de los cuales "lleva el nombre de unas de las naciones principales"; en cada calle las casas son iguales y representan las graciosas casas de los países extranjeros (citado por Benévolo, 1994: 105-106).

2. Ambos autores realizan propuestas de ciudades de gran envergadura.

Leonardo propone diez urbes enlazadas con una capacidad total de 50.000 viviendas y 300.000 habitantes aproximadamente, cifra nada desdeñable si la comparamos con los 125.000 habitantes con los que contaba la ciudad de Milán en el año 1483 (Zollner, 2007).

Por su parte, Cabet no establece un número de habitantes determinado para su nueva urbe, pero en la descripción de la ciudad de Icara utiliza dos términos que nos pueden sugerir el tamaño de la misma: "gran metrópoli" y "sesenta barrios" (citado por Benévolo, 1994: 105-106). Si consideramos que Cabet vivía en París y que en 1840 la urbe contaba con una población aproximada de un millón de habitantes, se puede considerar que pretendiera albergar en su nueva urbe al menos dicha población o incluso una mayor.

Con la descripción de Icara, se hacen evidentes las proporciones que pretendía:

Voyez, la ville presque circulaire, est partagée en deux parties à près égales par le Taïr (ou le Majeflueux) dont le cours a été redressé et enfermé entre deux murs en ligne presque droite, et dont le lit a été creusé pour recevoir les vaisseaux arrivant par la mer. Voilà le port, et les bassins, les magasins qui forment presque une ville entière! Vous voyez qu'au milieu de la ville, la rivière se divise en deux bras, que s'éloignent, se rapprochent et se réunissent à nouveau dans

\footnotetext{
9 "Véase la ciudad casi circular, partida en dos mitades bastante iguales por el Taïr (o el Majeflueux), cuyo curso fluvial ha sido redirigido y encerrado entre dos muros casi en línea recta y cuyo lecho fue dragado para recibir las embarcaciones que llegan por mar. He ahí el puerto y las dársenas, los almacenes que casi forman una ciudad entera. Verán que, en el centro de la ciudad, el río se divide en dos brazos, que se alejan, se acercan y se reúnen de nuevo en la dirección primitiva, de manera que forman una isla circular muy extensa. Esta isla es una plaza, la plaza central, poblada de árboles, en el centro de la cual se levanta un palacio que encierra un soberbio y vasto jardín, situado en una terraza elevada, en el centro del cual se eleva una enorme columna coronada por una estatua colosal que domina todos los edificios. Desde cada lado del río se percibe un ancho paseo, (avenida), bordeada por monumentos públicos. Alrededor de la plaza central y lejos de la misma, se pueden remarcar los dos círculos de otras plazas, uno de veinte y otro de cuarenta, casi igualmente alejados unos de otros y dispersos por toda la ciudad. iVéanse las calles, todas rectas y anchas! Cincuenta grandes atraviesan la ciudad, paralelas al río $y$ cincuenta la atraviesan perpendicularmente. Otras son más o
}

la direction primitive, de manière à former une île circulaire assez vaste. Cette île est un place, la place centrale, plantée d'arbres, au milieu de laquelle s'élève un palais enfermant un vaste te superbe jardin èlevé en terrasse, du centre duquel s'élance une immense colonne surmontée d'une statue colossale qui domine tous les édifices. De chaque côté de la rivière, vous apercevez un large quai bordé de monuments publics. Autour de cette place centrale et loin d'elle, vous pouvez remarquer deux cercles d'autres places, l'un de vingt et l'autre de quarante, presque également éloignées les unes des autres et dispersées dans toute la ville. Voyez les rues toutes droites et larges! En voilà cinquante grandes qui traversent la ville parallèlement á la rivière, et cinquante qui la traversent perpendiculairement. Les autres sont plus ou moins longues. Celles que vous voyez pointées en noir, et qui joignent ensemble les places, sont plantées d'arbres comme les boulevards de Paris. Les dix grandes rouges sont de rues de fer; toutes les jaunes sont des rues à ornières artificielles et les bleues sont des rues à canaux. Et qu'est-ce, lui demandai-je, que toutes ces larges et longues bandes roses que j'aperçois partout entre les maisons de deux rues?

Ce sont les jardins qui se trouvent sur le derrière de ces maisons. Je vous le montrerai tout à l'heure. Il y en a soixante; ce sont soixante quartiers, tous á peu près égaux, et représentant chacun l'étendue et la population d'une ville communale ordinaire. Chaque quartier porte le nom d'une des soixante principales villes du monde ancient et moderne, et présente dans ses nonuments et ses maisons l'architecture d'une des principales soixante nations 9 (Choay, 1965: 121-122).

En relación a la morfología y composición de cada uno de esos barrios, Cabet establece:

Voyons le plan d'un de ces quartiers! Tout ce qui est peint est edifice public. Voici l'école, l'hospital, le temple! Les rouges sont de grands ateliers, les jaunes sont de grands magasins, les bleus sont lex lieux d' assemblées, les violets sont les monuments. Remarquez que tous ces édificies public sont tellement distributes qu'il y en a dans toutes les rues, et que toutes les rues comprennent le meme nombre de maisons avec des édifices plus ou moins nombreux et plus ou moins vastes ${ }^{10}$ (Choay, 1965: 122).

menos largas. Las que se ven señaladas en negro que se juntan en las plazas, están plantadas de árboles como los bulevares de Paris. Las diez grandes, en rojo, son las vías férreas; todas las amarillas son las calles de carriles artificiales y las azules son canales. ¿Y qué son, le pregunté, todas esas anchas y largas bandas rosas que veo por todos lados, entre las casas y las calles? Son los jardines que se encuentran en la parte posterior de las casas. Se las mostraré más tarde. Hay sesenta barrios cada uno de los cuales lleva el nombre de una de las naciones principales del mundo antiguo y moderno, y representan las casas de la arquitectura de los países extranjeros [traducción de los autores].

${ }^{10}$ jY veamos el plano de uno de esos barrios! Todo lo que aparece coloreado son edificios públicos. La escuela, el hospicio, el templo. Los rojos son los grandes talleres, los amarillos, grandes almacenes, los azules son los lugares de asamblea y los violeta los monumentos. Observar que todos esos edificios públicos están distribuidos de tal forma que hay alguno en cada calle, y que todas las calles comprenden el mismo número de casa con unos edificios más o menos numerosos y grandes [traducción de los autores] 
Todo ello permite observar relevantes similitudes al considerar Leonardo el trazado de una gran urbe dividida en ciudades pequeñas que pueden encajar con la propuesta de sesenta barrios realizada por Cabet.

3. Son propuestas de urbes con separación de las vías de circulación.

En la Cittá idéale de Leonardo, las vías de comunicación y los canales están situados en dos niveles: el superior con calles abiertas y soleadas para el paso de personas y el inferior oscuro y cerrado para el tránsito de mercancías a las distintas partes de la Ciudad. Se encuentra ubicado bajo los patios de las viviendas y de los establos y está compuesto por vías de tránsito de carros y vías fluviales (Pedretti, 1988). Es el propio Leonardo quien lo confirma: "Por las calles altas no deben pasar carros, ni cosas semejantes, sino solamente los gentiles hombres. Por las calles bajas deberán ir los carros y otras bestias de carga, para uso y comodidad del pueblo. Cada casa deberá dar la espalda a otra, dejando la calle baja en medio" (citado por Franchetti, 1985: 570).

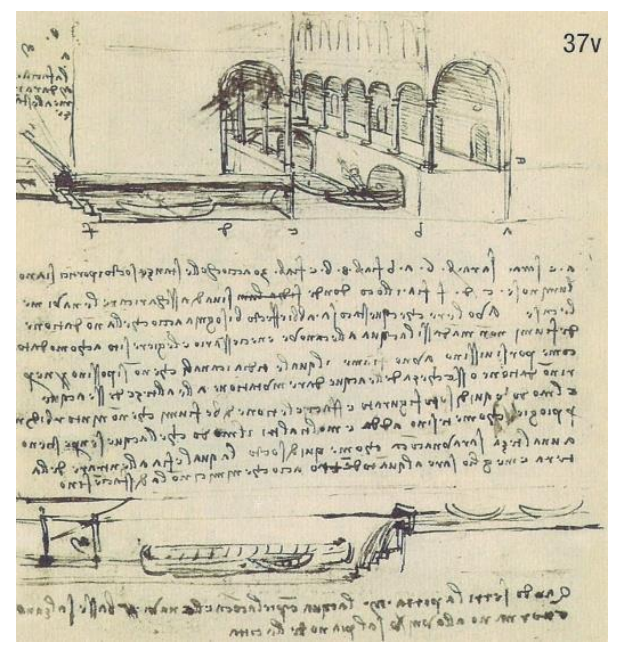

Figura 2: Descripción niveles de circulación.

Fuente: Manuscrito B, folio 37v. Biblioteca Ambrosiana da Vinci.

En el folio 37v del Manuscrito B (véase Figura 2) se puede apreciar parte de la fachada de un edificio que dispone de planta baja porticada. Los canales se sitúan en el nive inferior con túneles transversales que permiten la conexión con los almacenes del edificio. Con esta propuesta de circulación en dos niveles, es evidente el objetivo perseguido por Leonardo: garantizar las condiciones de higiene y salubridad de la población en las vías superiores, pues los desechos de los caballos, obras y mercancías transportadas se quedarían en el nivel

11 "Por las calles subterráneas inferiores discurra miseria, establos y lugares fétidos similares. De un arco a otro deben ser trecientos brazos, que cada calle reciba la luz de las rendijas de las calles de encima" [traducción de los autores].

12 "iContempla en primer lugar la construcción de las calles! Cada una tiene ocho carriles de hierro o de piedra para que circulen los coches de cuatro en fondo: dos pueden ir en un inferior, usando el curso fluvial soterrado como gigantesco albañal de evacuación.

Leonardo lo confirma con el siguiente comentario: "Per le vie socterane si de' votare destri, stalle e simile cose fetide. Dall' uno arco all'altro de' essere bracci trecento, cioè ciascuna via che riceve il lume dalle fessure delle strade di sopra"11 (Firpo, 1963: 75). Esta propuesta ha sido definida por la autora Helen Rosenau como "ciudad parcialmente subterránea" (1986: 62) en clara alusión a la disposición de circulación en dos niveles.

Por su parte, Cabet planteaba con claridad, la separación de la circulación de Icara en ocho carriles: para carruajes, para máquinas con ruedas y por supuesto para los peatones. La diferencia con respecto a la propuesta de Leonardo radica en que todos los carriles se encuentran al mismo nivel, no utiliza en su propuesta el subsuelo de la Ciudad.

Vois d' abord la construction des rues! Chacune a huit ornières en fer ou en pierre pour quatre voitures de front, dont deux peuvent aller dans un sens et deux dans un autre. Les roues ne quittent jamais ces ornières, et les cevaux ne quittent jamais le trottoir intermédiaire. Les quatro trottoirs sont pavés en pierres ou cailloux, et toutes les autres bandes de la rue sont pavées en briques. Les roues ne Font ni boue ni poussieère, les vhevaux n'en Font presque point, les machines n'en Font pas du tout sur les rues-chemins-defer ${ }^{12}$ (Choay, 1965: 125).

4. Ambos autores establecen un procedimiento de limpieza de las calles.

En la propuesta de Leonardo, el vial de nivel superior presenta una pendiente hacia el centro del mismo, lugar donde se ubican unas rejillas que permiten la iluminación y la evacuación de las aguas de lluvia y riego hacia el nivel inferior. En este último (véase Figura 3) ocurre lo mismo y la evacuación se realiza mediante rejillas centrales hacia el subsuelo (Firpo, 1963) con respecto a la evacuación de residuos de las viviendas, pues los canales del nivel inferior circulan bajo las viviendas, permitiéndose la descarga directa de los desperdicios.

También a través de los canales se contempla la posibilidad de llevar mediante barcas las mercancías hasta el nivel inferior de las viviendas. En ningún caso se explica si habría canales separativos para los residuos. Es interesante como Leonardo propone que los pórticos de las viviendas se sitúen directamente sobre los canales, para así poder acceder hasta el interior del edificio. Seguramente, en un nivel arquitectónico, pensó en viviendas con escaleras hasta el sótano, de tal forma que se pudiera acceder hasta los canales (Pedretti, 1988).

sentido y dos en el otro. Las ruedas no salen nunca de esos carriles y los caballos no salen nunca de la acera intermedia. Las cuatro aceras están empedradas con piedras o guijarros, y las demás bandas de la calle están soladas con ladrillos. Las ruedas no forman barro, ni levantan polvo, ni tampoco las máquinas que van sobre rueda, como el tren..." [traducción de los autores]. 


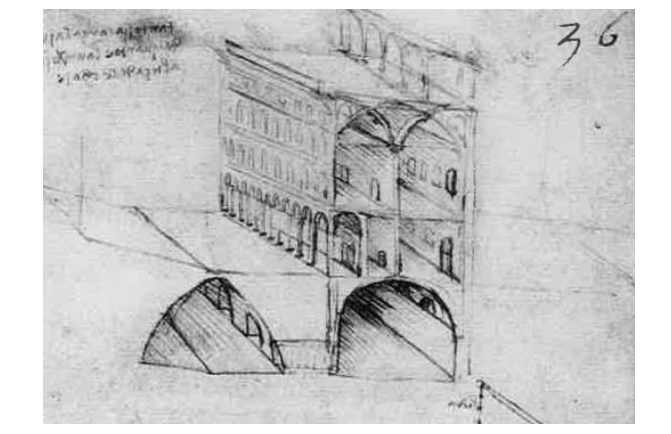

Figura 3: Descripción niveles de circulación Fuente: Manuscrito B, folio 36r. Biblioteca Ambrosiana da Vinci.

Por su parte, Cabet establece una serie de instrucciones que garantizan la higiene y la salubridad de las calles, tales como: no tirar basura a la calle, no transportar paja, heno ni estiércol por la Ciudad porque todas las instalaciones que usan estos productos se encuentran ubicadas a las afueras de la misma. También la obligación del cierre hermético de las mercancías de los carros, estableciendo un procedimiento mecánico para la carga y descarga que no ensucia (Choay, 1970); no obstante, en caso de necesitar limpiar las calles, Cabet contempló el siguiente proceso:

Nunca podré indicarte las precauciones que se han tomado para la limpieza de las calles. Es muy sencillo que las aceras se barran y rieguen todas las mañanas, y que siempre estén perfectamente limpias; las calles están adoquinadas construidas de tal manera que las aguas no se estancan, ya que a cada paso encuentran aberturas por donde escapar a unos canales subterráneas. El barro, recogido y barrido con ayuda de instrumentos ingeniosos y cómodos, no solo es arrastrado a los mismos canales por las aguas de las fuentes, sino que emplean todos los medios que puedas imaginar para que se forme la menor cantidad de barro y polvo (citado por Choay, 1970: 156-157).

Por tanto, la descripción del proceso de limpieza de las calles de Icaria es, salvando los procesos tecnológicos, igual a la que realiza Leonardo para su ciudad ideal 350 años antes.

5. Contemplan la ventilación y la salubridad de las viviendas.

Leonardo establece la necesidad de ventilación e iluminación en el interior de las viviendas; por ello, cuentan con ventanas abiertas a las calles. Las dimensiones de éstas también se encuentran reguladas, ${ }^{13}$ de tal forma que su anchura es igual o mayor a la altura de los edificios. ${ }^{14}$ También es necesario disponer de instalaciones de abastecimiento y saneamiento en las viviendas, garantizadas gracias a los canales ubicados a nivel inferior. En la figura 4,

13 Estas indicaciones deben ser consideradas como un precedente a las teorías higienistas que surgieron posteriormente durante el siglo XIX. correspondiente al folio $47 \mathrm{r}$ del Manuscrito B, se aprecia cómo el bloque de escaleras del edificio se encuentra en la parte central y Leonardo refleja la distribución de una vivienda (Pedretti, 1988). El resto del edificio es representado mediante líneas esquemáticas debido a la similitud en la distribución de todas las viviendas; destaca de la figura el ingenioso sistema de escaleras dobles que permiten el acceso a cada vivienda de forma independiente.

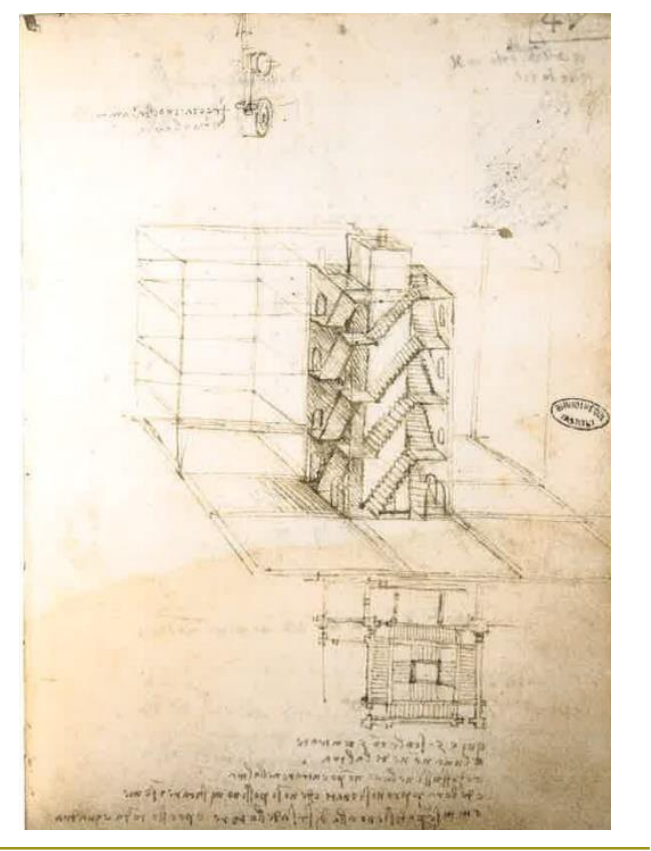

Figura 4: Escaleras de edificio en Cittá idèale. Fuente: Manuscrito B, folio 47r. Biblioteca Ambrosiana da Vinci.

La descripción que realiza Cabet de las viviendas para su nueva urbe es la siguiente, (citado por Choay, 1970: 160161):

Cada casa tiene cuatro pisos, sin contar con la planta baja; $y$ tres o cuatro o cinco ventanas a todo lo ancho. La planta baja está situada sobre los sótanos, las leñeras y las carboneras cuya base se encuentra a cinco o seis pies por debajo de la acera y la bóveda a tres o cuatro pies por encima. La madera, el carbón y todo lo demás se transporta de los coches a las piezas subterráneas por medio de máquinas, sin tocar siquiera la acera. Luego, todos estos objetos se suben en cestos o vasijas a la cocina y pisos superiores, a través de unos boquetes abiertos en la bóveda [...] ¡Que hermosa vista! exclamé al llegar a una terraza, bordeada de una balaustrada y cubierta de flores que corona la casa y forma incluso un jardín delicioso y distinto desde el que hay una vista magnifica [...] Las aguas potables se distribuyen por medio de tubos y grifos por todas las plantas y por todos los pisos. Todas las inmundicias y aguas sucias son arrastradas, sin que queden estancadas ni quede

universale altezza delle case" (Cantone, 1978: 79) / "Por lo que el camino sea ancho, ya que la altura de las casas es universal" [traducción de los autores] 
mal olor, a unos anchos tubos subterráneos situados bajo las calles.

Es indiscutible que ambos autores profesan una fuerte preocupación por la salubridad e higiene de las viviendas, pues cuentan con instalaciones de abastecimiento, evacuación de aguas residuales y zona de recepción de mercancías a nivel de sótano. No obstante, se observa alguna diferencia al respecto: mientras Cabet establece que cada casa de cuatro pisos albergue a una familia, Leonardo distribuía una familia por piso. De su planteamiento se deducen las diferencias de tamaño aludidas anteriormente entre la población de ambas ciudades.

\section{Conclusiones}

De sus estudios se deduce el interés de Leonardo por la arquitectura y el urbanismo; prueba de ello se encuentra en su propuesta de Cittá idèale, considerada como excepcional y de una brillantez majestuosa. Se adelanta 400 años a su época y le otorga el reconocimiento de ser un urbanista excepcional. Luigi Firpo lo califica como: "è veramente il primo urbanista moderno" 15 (Firpo, 1963: 64), que implica considerar a Leonardo como el promotor del cambio del urbanismo de la época, posibilitando el desarrollo del urbanismo moderno.

Son evidentes y sorprendentes las similitudes existentes entre la propuesta de Cittá idéale de Leonardo y la descrita por Étienne Cabet en su publicación Voyage en Icarie, teniendo en cuenta que entre ambas existe una diferencia temporal de aproximadamente 350 años -la

\section{Bibliografía}

Benévolo, L. La captura del infinito. Madrid, España: Ediciones Celeste, 1994.

Benévolo, L. Historia de la arquitectura moderna. Barcelona: Editorial Gustavo Gili, 2007.

- Cabet, É. Voyage en Icarie 1840. Slatsine, París. Edición consultada: Viaje por Icaria. Barcelona: Editorial Orbis, 1985

- Cantone, G. La citta di Marmo: da Alberti a Serlio. La storia tra progettazione e restauro. Roma: Officina Edizioni, 1978.

- Choay, F. L' urbanisme, utopies et réalités. París: Éditions du Seuil, 1965.

- Choay, F. El urbanismo: utopías y realidades. Barcelona: Editorial Lumne, 1970.

- Firpo, L. Leonardo architetto e urbanista. Turín Unione tipográfico \& Editrice Tourinese, 1963.

- Franchetti, V. Historia del urbanismo: siglos XIV y $X V$. Madrid: Instituto de estudios de administración local, 1985. propuesta de Leonardo es de 1485 y la de Cabet de 1840-. Durante este periodo de tiempo se produjo una evolución en la tecnología, circunstancia que se hace evidente en la descripción que ambos autores hacen de sus propuestas; no obstante, los principios fundamentales de desarrollo de ambas ideas son similares.

Se desconocen las teorías o propuestas en las que se basó Cabet para el desarrollo de su idea, aunque es posible que conociera el proyecto de Leonardo que se encontraba, desde 1795, en la biblioteca del Institut de France en Paris que no cuenta con un registro de lectores del "Manuscrito B" de Leonardo que nos permita asegurar, el conocimiento del contenido del mismo por parte de Étienne Cabet; sin embargo, las similitudes descritas nos llevan a considerar que ese conocimiento pudo haberse producido.

Con independencia de esto, ambas propuestas son magníficas y deben ser consideradas precursoras dentro del campo de la salubridad, si consideramos que a mediados del siglo XIX empezaba a desarrollarse.

Como citar este artículo/How to cite this article: Hidalgo, D., Santiago, J., y Arco, J. (2015). Desarrollos urbanos paralelos: Leonardo Da Vinci - Étienne Cabet. Estoa, Revista de la Facultad de Arquitectura y Urbanismo de la Universidad de Cuenca, 4(7), 7-13. doi:10.18537/est.v004.n007.02
García, N. "Los Códices de Leonardo en España". Boletín del Seminario de Arte y Arqueología 63 (2010): 371-395.

Garín, E. El Renacimiento italiano. Barcelona: Editorial Ariel, 1982.

Pedretti, Carlo. Leonardo architetto. Milán: Ediciones Electa, 1988.

Rosenau, H. La ciudad ideal, su evolución arquitectónica en Europa. Madrid: Editorial Alianza, 1986

Vinci, L. Manuscritos de Francia 1485. París: Institut de France. Edición consultada: Facsímil de la Biblioteca Ambrosiana de Vinci. Florencia, 2012.

Zollner, F. Leonardo da Vinci, obra pictórica completa y obra gráfica. Madrid: Editorial Taschen, 2007. 